\title{
Classificação das periodontites em indivíduos jovens - revisão da literatura e relato de casos clínicos
}

\author{
HAAS, Alex Nogueira ${ }_{*}^{*}$ \\ MORENO, Tatiana* \\ RÖSING, Cassiano Kuchenbecker
}

\begin{abstract}
RESUMO
Inúmeros sistemas classificatórios das doenças periodontais têm sido sugeridos ao longo das décadas. Particularmente, a classificação das periodontites em indivíduos jovens tem recebido especial atenção devido a recentes alterações propostas pela Academia Americana de Periodontia em 1999, alterando nomenclaturas anteriormente utilizadas por um período longo de tempo. Assim sendo, a presente revisão de literatura teve como objetivo descrever e discutir alguns termos de classificações utilizadas na literatura para as periodontites em indivíduos jovens. Além disso, descrever casos clínicos de periodontites em jovens a fim de ilustrar possíveis classificações nesse tipo de paciente.
\end{abstract}

\section{PALAVRAS-CHAVE: \\ Periodontite. Periodontite agressiva, diagnóstico.}

\section{INTRODUÇÃO}

As doenças periodontais são caracterizadas por serem patologias infecto-inflamatórias causadas por bactérias presentes nos biofilmes dentais e podem afetar indivíduos de todas as idades (LOE et al., 1986). Elas são divididas em dois grandes grupos de doenças: gengivites e periodontites. As gengivites são doenças que afetam o periodonto marginal e são causadas pelo biofilme supragengival (LOE et al., 1965). As periodontites são doenças destrutivas que acometem indivíduos suscetíveis, de tal forma a gerar perda de suporte periodontal (ligamento periodontal e osso alveolar) decorrente do acúmulo de biofilme subgengival (LINDHE et al. 1975). Essa divisão das doenças periodontais, contudo, é ampla e requer uma visão mais específica, tendo em vista a existência de diferentes formas em que estas doenças podem se apresentar nas populações (ALBANDAR, 2002). Neste sentido, ao longo das décadas, inúmeros sistemas classificatórios têm sido sugeridos a fim de facilitar o diagnóstico e universalizar a nomenclatura em nível acadêmico, clínico e epidemiológico (ARMITAGE, 2002). No que se refere às periodontites em indivíduos jovens, a variação na nomenclatura tem recebido es- pecial atenção.

Dessa forma, o objetivo do presente relato foi buscar na literatura a nomenclatura utilizada por diferentes classificações das periodontites em indivíduos jovens, bem como descrever três casos clínicos de diferentes formas de periodontite em jovens.

\section{REVISÃO DA LITERATURA}

Historicamente, os termos utilizados para descrever as periodontites em indivíduos jovens evoluíram de acordo com o conhecimento da etiologia das doenças periodontais. Gottlieb et al., ainda em 1923, descreveram as periodontites em jovens como "atrofia difusa do osso alveolar", tendo em vista que essa era a visão da época sobre as causas das doenças gengivais. Mais tarde, o que se acreditava ser um processo degenerativo, foi denominado "periodontosis", e seria causada por fatores não-locais. $O$ termo "periodontosis" foi fortalecido por Baer (1971), o qual, em seu artigo, a doença foi dividida em duas categorias: a forma localizada que afetaria os primeiros molares e incisivos, e a forma generalizada que afetaria a maior parte da dentição. O termo "periodontite juvenil", introduzido por Butler (1969), descrevia a do- ença como um processo inflamatório resultante da infecção do periodonto por bactérias da cavidade bucal. Waerhaug, em 1977, veio a embasar o conceito da placa bacteriana como fator causal das doenças periodontais, descrevendo a placa como etiologia primária da doença, incluindo também as periodontites em jovens.

Inúmeros critérios também foram sugeridos para guiar a classificação das doenças periodontais destrutivas. O Consensus Report do Workshop Mundial de Periodontia Clínica de 1989 usou os seguintes critérios para distinguir as várias formas de periodontite: (1) idade de início, (2) taxa de progressão da doença, (3) distribuição dos sítios afetados, (4) presença ou ausência de condições médicas sistêmicas, (5) presença ou ausência de microrganismos específicos ou fatores do hospedeiro, e (6) resposta à terapia (American Academy of Periodontology, 1989). Entretanto, tais critérios continuam sendo discutidos e têm gerado controvérsia quanto a sua real validade. Mesmo assim, com base nestes critérios de classificação, as doenças periodontais em indivíduos jovens receberam, naquela época, a denominação de Periodontites de Estabelecimento Precoce, que po-

* Mestre e doutorando em Odontologia-Periodontia pela UFRGS; Professor Assistente de Clínica Odontológica da Faculdade de Odontologia da UFRGS.

** Mestre em Odontologia-Periodontia pela UFRGS. *** Doutor em Periodontia pela Faculdade de Odontologia de Araraquara da Universidade Estadual Paulista Júlio de Mesquita Filho; Professor de Periodontia da ULBRA-Canoas e da Faculdade de Odontologia da UFRGS. 
deriam ser subdivididas em: Periodontite Pré-Puberal (localizada ou generalizada), Periodontite Juvenil (localizada ou generalizada) e Periodontite de Progressão Rápida.

A Periodontite Pré-Puberal foi definida como a doença periodontal que ocorre durante ou logo após o irrompimento dos dentes decíduos. Foi descrita como uma condição rara e, por este motivo, não existem definições de nível de perda de inserção e de números de dentes envolvidos. Segundo Page et al. (1983), a forma localizada atinge alguns dentes da dentição decídua, mais comumente os molares. A quantidade de placa associada é moderada e há pouca inflamação gengival, mas existindo sangramento à sondagem em sítios afetados. Não existem condições sistêmicas associadas, e a progressão é moderada. A forma generalizada afeta todos os dentes decíduos, podendo causar sua exfoliação precoce. Apresenta progressão rápida e inflamação dos tecidos gengivais.

A Periodontite Juvenil seria aquela doença que ocorre em crianças e adolescentes sistemicamente saudáveis, sendo caracterizada por rápida destruição do osso alveolar. A forma localizada, segundo Baer, é caracterizada por perda de inserção de $3 \mathrm{~mm}$ ou mais em pelo menos dois primeiros molares e incisivos (pelo menos um primeiro molar deve estar afetado), e perda de inserção maior que 3 mm em não mais que dois dentes além dos primeiros molares e incisivos. Apresenta uma tendência à agregação familiar, e a idade de início seria entre 25 a 30 anos. Pacientes apresentando perda de inserção em mais de dois dentes além dos primeiros molares e incisivos, são classificados no grupo de Periodontite Juvenil Generalizada, que apresentaria uma idade de início da doença um pouco mais elevada, porém antes dos 35 anos. $\mathrm{O}$ grupo de Periodontite Juvenil Generalizada (PJG) é um grupo mais heterogêneo, que inclui formas mais graves de periodontite e Periodontite de Progressão Rápida, esta última não apresentando características suficientemente diferentes das atribuídas à PJG para se constituir um outro grupo (BAER, 1971; RANNEY, 1992; TONETTI; MOMBELLI, 1999).

No entanto, esta classificação apresenta alguns pontos questionáveis. Uma questão relevante é o uso da idade como um descritor primário para a doença. Este fato leva à idéia de que pacientes diagnosticados com base na idade teriam seu diagnóstico modificado quando ficassem mais velhos, mesmo apresentando a mesma doença, do ponto de vista etioló- gico e de suscetibilidade. Além disso, usar a idade como critério de classificação é limitado pelo fato de a idade, no momento do diagnóstico, não ser necessariamente a mesma do aparecimento da doença e, assim, tornando-se impossível precisar o exato momento do início do processo de destruição (ARMITAGE, 1999; ARMITAGE, 2002; TONETTI, MOMBELLI, 1999).

Outro ponto questionável é a utilização de critérios como a distribuição das lesões, caracterizando a doença como localizada ou generalizada, já que estudos têm demonstrado que a doença localizada pode converter-se em generalizada, se tratamento apropriado não é realizado (Albandar et al., 1997; Burmeister et al., 1984). Outro aspecto é a taxa de progressão, visto que não é possível determinála, a não ser em observações longitudinais do caso (ARMITAGE, 1999; ARMITAGE, 2002; TONETTI; MOMBELLI, 1999).

Baseados no conhecimento destas falhas do sistema de classificação de 1989, uma nova classificação foi proposta, em 1999, pela Academia Americana de Periodontia (AAP). $O$ termo Periodontite Pré-Puberal foi colocado em desuso, visto que a maior parte dos casos generalizados desta condição estava relacionada a doenças sistêmicas. Já os casos não relacionados às desordens sistêmicas foram re-alocados nos grupos de periodontite crônica ou agressiva, dependendo das características de cada caso, visto que a idade deixou de ser fator primário para a classificação (ARMITAGE, 1999). No modelo proposto, a Periodontite de Estabelecimento Precoce é agora denominada Periodontite Agressiva, que pode ser localizada ou generalizada (AMERICAN ACADEMY OF PERIODONTOLOGY, 1999; AMERICAN ACADEMY OF PERIODONTOLOGY, 2000).

Os fatores primários e comuns às formas localizada e generalizada de periodontite agressiva são:

- pacientes sistemicamente saudáveis; - rápida perda de inserção e destruição de osso alveolar;

- agregação familiar.

Fatores secundários que estão geralmente presentes (mas não necessariamente em todos os casos) são:

- quantidades de depósitos microbianos inconsistentes com a severidade de destruição periodontal;

- elevadas proporções de Actinobacillus actinomycetemcomitans $\mathrm{e}$, em algumas populações, Porphyromonas gingivalis podem estar presentes;

- anormalidades fagocitárias;
- macrófagos hiper responsivos; incluindo níveis elevados de citocinas;

- progressão da perda de inserção e perda óssea podem ser auto-limitantes.

Nem todas estas características necessitam estar presentes para diagnosticar ou classificar a doença. $O$ diagnóstico deve ser baseado principalmente nos dados clínicos e história do paciente obtida através da entrevista (AMERICAN ACADEMY OF PERIODONTOLOGY, 1999; AMERICAN ACADEMY OF PERIODONTOLOGY, 2003). Dados radiográficos são secundários ao diagnóstico e à classificação.

Alguns destes fatores que caracterizariam a periodontite agressiva têm sido questionados. Classicamente, e ainda na nova classificação da AAP, considera-se que pacientes com periodontite agressiva apresentariam pequena quantidade de placa bacteriana e cálculo (BAER, 1971). Em contraste, investigações mais recentes demonstraram níveis de placa e cálculo em pacientes com periodontite agressiva semelhantes àqueles relatados para as formas crônicas de periodontite, ou inclusive maiores (BURMEISTER et al., 1984; ALBANDAR et al., 1996; SUSIN; ALBANDAR, 2005). Ainda, segundo o Workshop Internacional para a Classificação das Doenças e Condições Periodontais (AMERICAN ACADEMY OF PERIODONTOLOGY, 1999), crianças e adolescentes podem apresentar qualquer uma das formas de periodontite.

A periodontite crônica é mais prevalente em adultos, mas pode ocorrer em crianças e adolescentes. Existem evidências de que, em algumas populações, a destruição periodontal tem início precoce na vida dos indivíduos. Pode ser localizada (afetando menos que $30 \%$ da dentição) ou generalizada (afetando mais que $30 \%$ da dentição) e é caracterizada por uma taxa de progressão lenta ou moderada, que pode incluir períodos de rápida destruição. Além disso, a severidade da doença pode ser leve (1 a $2 \mathrm{~mm}$ de perda de inserção), moderada (3 a 4 mm de perda de inserção) ou severa ( ${ }^{3} 5 \mathrm{~mm}$ de perda de inserção). Crianças e adultos jovens com essa forma de doença eram anteriormente estudados juntamente com pacientes que apresentavam periodontite agressiva localizada ou generalizada. Entretanto, faltam estudos publicados sobre este grupo (AMERICAN ACADEMY OF PERIODONTOLOGY, 2003; VAN DER VELDEN, 1991)

Perda de inserção incidental é um termo utilizado como um descritor epidemiológico usado para situações onde 
a perda de inserção clínica não pode ser atribuída a doenças periodontais como a periodontite crônica ou agressiva. Löe e Brown realizaram um estudo epidemiológico de periodontite em indivíduos entre 14 e 17 anos nos Estados Unidos. Em virtude da baixa idade dos indivíduos da amostra, perda de inserção ${ }^{3} 3$ mm foi utilizada como critério para diagnóstico de periodontite ou perda de inserção incidental. Foi encontrado que $71 \%$ dos indivíduos do grupo de perda de inserção incidental apresentavam um sítio afetado e $97 \%$ dos indivíduos possuíam 3 ou menos sítios afetados. Esta definição é uma maneira de classificar aqueles indivíduos que possuem poucos sítios afetados por perda de inserção anormal, ou seja, não associada com causas locais como cáries proximais ou restaurações mal adaptadas (ALBANDAR et al., 1997; KINANE, 2001; LÖE; BROWN, 1991; AMERICAN ACADEMY OF PERIODONTOLOGY, 2003). Esta perda de inserção incidental em alguns indivíduos pode progredir para uma forma avançada de periodontite. Nestes casos, esta perda de inserção poderia ser considerada periodontite incipiente ou inicial. Os pacientes com este diagnóstico clínico devem ser considerados como um grupo de risco para o desenvolvimento de doença periodontal, visto que, no estudo longitudinal de Albandar, um quarto dos pacientes que apresentavam perda de inserção incidental apresentou posteriormente progressão moderada ou rápida da doença (ALBANDAR et al., 1997).

\section{CASOS CLÍNICOS}

Caso clínico 1
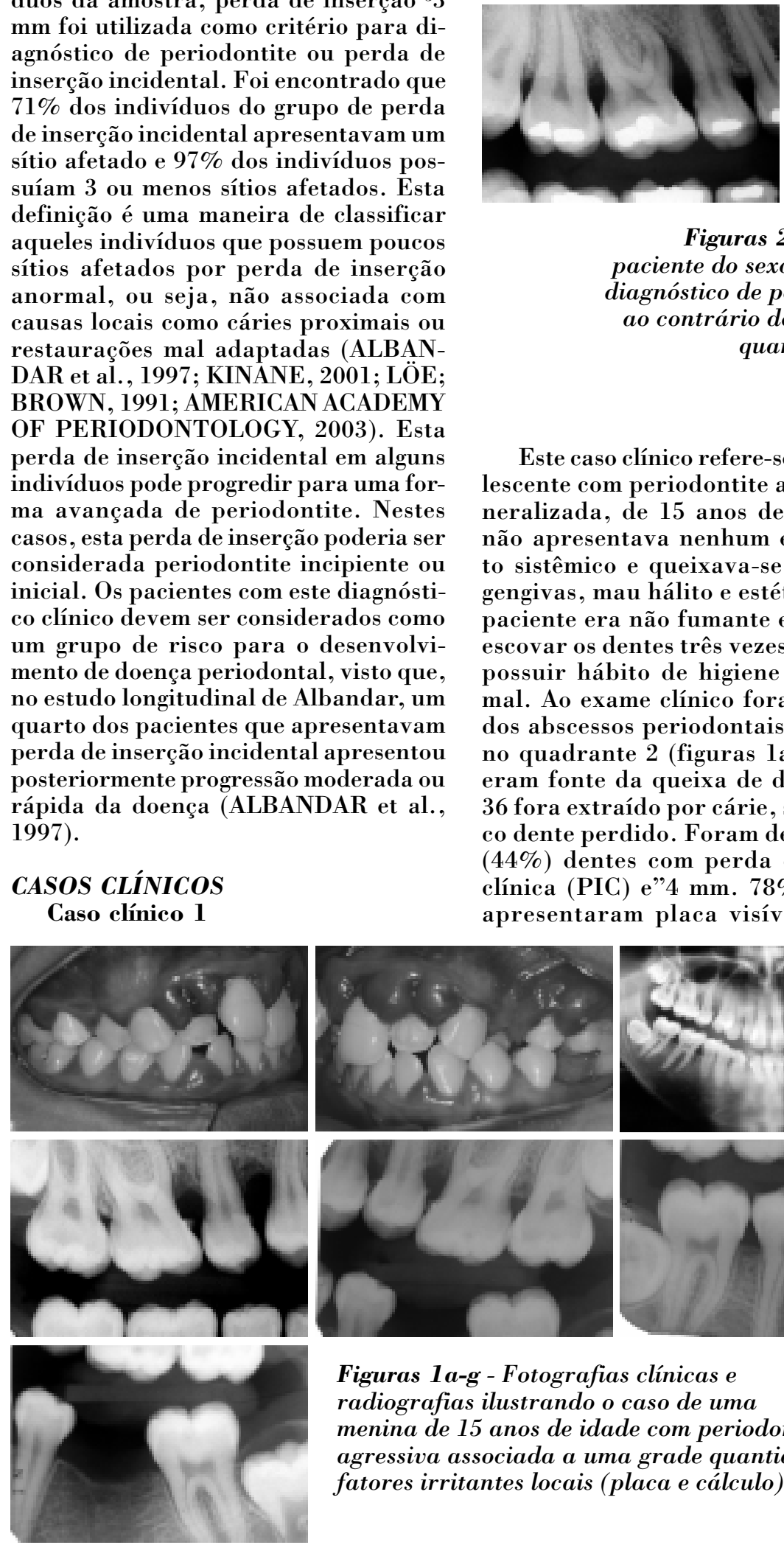

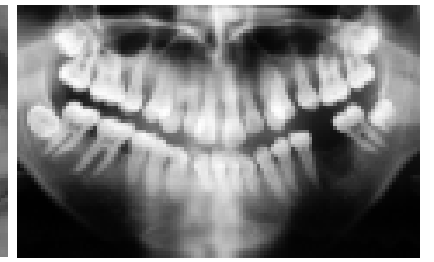

Figuras 1a-g - Fotografias clínicas e radiografias ilustrando o caso de uma menina de 15 anos de idade com periodontite agressiva associada a uma grade quantidade de fatores irritantes locais (placa e cálculo).

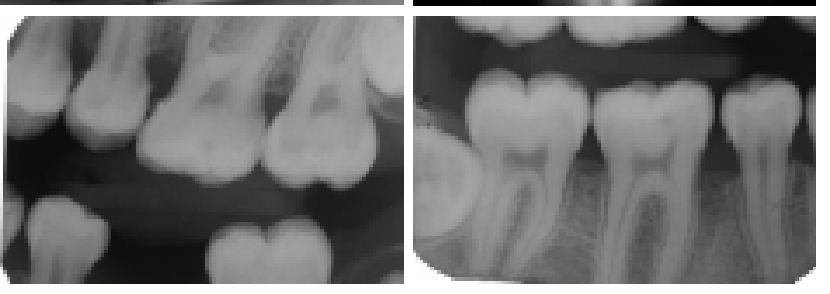

Este caso clínico refere-se a uma adolescente com periodontite agressiva generalizada, de 15 anos de idade, que não apresentava nenhum envolvimenortemico e queixava-se de dor nas gengivas, mau hálito e estética ruim. A paciente era não fumante e costumava covar os dentes três vezes ao dia, sem mal. Ao exame clínico foram observaeram fonte da queixa de dor. $O$ dente 36 fora extraído por cárie, sendo o único dente perdido. Foram detectados 12 (44\%) dentes com perda de inserção clínica (PIC) e"4 mm. 78\% dos sítios apresentaram placa visível (ÍNDICE

fatores irritantes locais (placa e calculo).
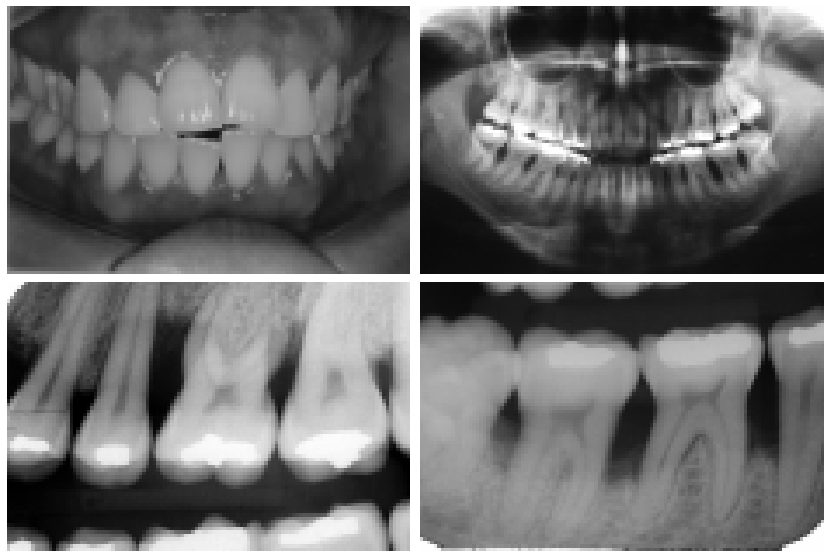

Figuras 2a-g-Caso clínico de uma paciente do sexo feminino de 16 anos com diagnóstico de periodontite agressiva que, ao contrário do caso 1 , apresenta pouca quantidade de placa e cálculo supragengivais.

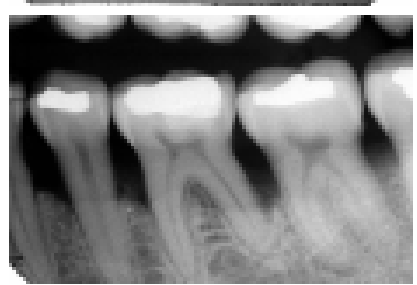

DE PLACA VISÍVEL - IPV - AINAMO E BAY, 1975) e 40\% possuíam sangramento gengival (ÍNDICE DE SANGRAMENTO GENGIVAL - ISG - AINAMO E BAY, 1975). A presença de cálculo foi um achado em $45 \%$ dos sítios. As médias de profundidade de sondagem (PS) e PIC eram de 4,3 $\mathrm{mm}$ e 1,6 $\mathrm{mm}$, respectivamente, sendo que $17 \%$ e $49 \%$ dos sítios apresentavam PS e PIC e"4 mm. Radiograficamente, observam-se perdas ósseas extensas em primeiros molares, incisivos e pré-molares (figuras $1 c-g)$.

\section{Caso clínico 2}

Paciente do sexo feminino, 26 anos de idade, não fumante, com periodontite agressiva generalizada, buscou atendimento por indicação de ortodontista. Durante a entrevista não foram detectadas doenças sistêmicas. Além disso, a paciente relatou uso constante de fio dental e escova dental macia, porém nunca havia recebido tratamento periodontal subgengival, apenas sessões de controle de placa supragengival profissional. Dos 28 dentes presentes, 22 estavam afetados pela doença com PIC e"4 mm. Os percentuais de IPV, ISG e cálculo foram 35\%, $5 \%$ e $10 \%$, respectivamente, o que demonstrou um razoável controle da placa supragengival, em contraste com a severidade da doença (médias de PS e PIC de 4,3 $\mathrm{mm}$ e $3,2 \mathrm{~mm}$, respectivamente). A figura $2 b$ ilustra o grau de perda de inserção clínica na mesial do dente 31 . Quanto à extensão da doença, quase a metade dos sítios apresentaram PS e PI com $4 \mathrm{~mm}$ ou mais $(48 \%$ e $41 \%$, res- 
pectivamente), sendo a perda óssea presente em todos os dentes, com pouca expressão apenas em incisivos superiores, como pode ser visto na radiografia panorâmica da figura 2c (Figuras $2 \mathrm{a}-2 \mathrm{~g})$.

\section{Caso clínico 3}

Indivíduo do sexo masculino, de 13 anos de idade, não fumante, foi levado por sua mãe para exame de diagnóstico periodontal, pois esta foi informada durante tratamento periodontal sobre os aspectos genéticos das periodontites. Ao exame clínico, foram observados $59 \%$ e $11 \%$ dos sítios com placa e gengivite. Cálculo era quase inexistente supragengivalmente. Apenas o dente 46 apresentava $P S=6 \mathrm{~mm}$ e $P I C=4 \mathrm{~mm}$ na face distal por lingual (figura $3 b$ ), com sangramento à sondagem subgengival. A partir dos dados clínicos e da entrevista, foi dado diagnóstico de perda de inserção incidental. Radiograficamente, o paciente apresentava perda óssea localizada nos primeiros molares inferiores (figuras 3c-d)

\section{CONSIDERACŨES FINAIS}

Uma classificação ideal para as doenças periodontais que acometem indivíduos jovens deveria ser baseada em fatores etiológicos e na suscetibilidade do indivíduo, e a partir disso, embasar um tratamento focado e de prognóstico preciso. Á luz dos conhecimentos atuais, ainda não é possível propor este tipo de classificação (ARMITAGE, 1999; TONETTI, MOMBELLI, 1999). Os sistemas de classificação são importantes para proporcionar uma estrutura para estudos científicos sobre etiologia, patogênese e tratamento das doenças de maneira organizada, e também dar aos clínicos um guia para estabelecer os cuidados de saúde necessários para seus pacientes. Porém, mais importante do que a classificação das doenças, é reconhecer que cada paciente tem a "sua" doença, que eventualmente se encaixa em algum item da classificação, mas que cada indivíduo tem os seus fatores de suscetibilidade genéticos, comportamentais e ambientais, e estes são os fatores de fato relevantes, que irão proporcionar um adequado diagnóstico e tratamento da condição deste indivíduo. Ainda, há que se reconhecer que o maior impacto que as doenças periodontais têm quando acometem indivíduos jovens é que a seqüela da doença (perda de inserção) apresenta caráter cumulativo, podendo levar ao desfecho real da doença (perda dentária) muito precocemente na vida do indivíduo.
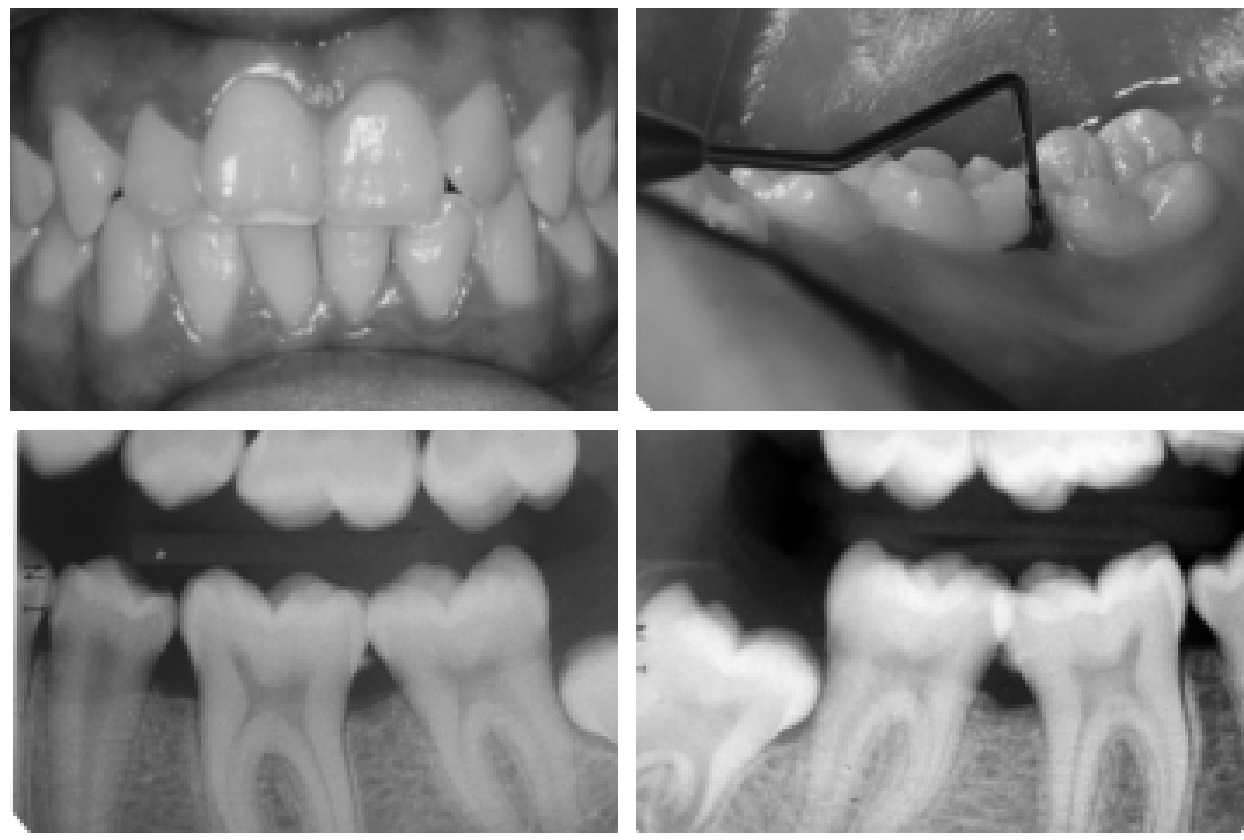

Figuras 3a-d - Perda de inserção incidental em paciente do sexo masculino de 13 nos de idade com história familiar de periodontite.

\section{ABSTRACT}

A significant number of classificatory systems of periodontal diseases have been suggested during the past decades. The classification of periodontitis in young individuals, particularly, has received special attention due to recent modifications made by the American Academy of Periodontology in 1999, changing previous nomenclature used by a long period of time. Thus, the aim of this literature review was to describe and discuss some classificatory terms found in the literature to classify periodontitis in young persons. Additionally, to describe case reports of periodontitis in young individuals aiming at illustrating possible classifications in this patients.

\section{KEY WORDS:}

Periodontitis. Aggressive periodontitis, diagnosis.

\section{REFERÊNCIAS}

AINAMO, J, BAY, I. Problems and Proposals for Recording Gingivitis and Plaque. Int. Dent. J., v.25, no.4, p.229-235, 1975.

ALBANDAR, J.M. et al., Gingival State and Dental Calculus in Early-Onset Periodontitis. J Periodontol, Chicago, v.67, p.953-959, 1996.

ALBANDAR, J.M. et al. Clinical Classification of Periodontitis in Adolescents and Young Adults. J Periodontol, Chicago, v.68, p. 545-555, 1997.
ALBANDAR, J.M. Global Risk Factors and Risk Indicators for Periodontal Diseases.

Periodontol 2000, Copenhagen, v.29, p.177-206, 2002.

AMERICAN ACADEMY OF PERIODONTOLOGY. Consensus Report: Aggressive Periodontitis. Ann Periodontol, Chicago, v.4, p.53, 1999.

AMERICAN ACADEMY OF PERIODONTOLOGY. Parameters of Care Supplement - Parameter on Agressive Periodontitis. J Periodontol, Chicago, v.71, suppl, p. 867-869, 2000.

AMERICAN ACADEMY OF PERIODONTOLOGY. Position Paper - Periodontal Diseases of Children and Adolescents. J Periodontol, Chicago, v.74, p. 1696-1704, 2003.

AMERICAN ACADEMY OF PERIODONTOLOGY. Proceedings of the World Workshop in Clinical Periodontics. Chigago, 1989.

ARMITAGE, G.C. Development of a Classification System for Periodontal Diseases and Conditions. Ann. Periodontol., Chicago, v.4, p. 1-6, 1999.

ARMITAGE, G.C., Classifying Periodontal Diseases - A Long-Standing Dilemma. Periodontol 2000, Copenhagen, v.30, p. 9-23, 2002. 
BAER, P.N. The Case for Periodontosis as a Clinical Entity. J. Periodontol., Chicago, v.42, p. 516-520, 1971.

BURMEISTER, J.A. et al., Localized Juvenile Periodontitis and Generalized Severe Periodontitis: Clinical Findings. J. Clin. Periodontol., Copenhagen, v.11, p.181-192, 1984.

BUTLER, J.H. A Familial Pattern of Juvenile Periodontitis (periodontosis).

J. Periodontol., Chicago, v.40, $\mathrm{n}^{\circ} .2$, p.115-118, 1969.

KINANE, D.F. Periodontal Disease in Children and Adolescents: Introduction and Classification. Periodontol. 2000, Copenhagen, v.26, p. 7-15, 2001.

LINDHE, J. et al. Experimental Periodontitis in the Beagle Dog. J. Periodont. Res., Copenhagen, v.8, p.1-10, 1973.

LÖE, H. et al. Experimental Gingivitis in Man. J. Periodontol., Chicago, v.36, p.177-87, 1965.
LÖE, H. et al. The Natural History of Periodontal Disease in Man. Rapid, Moderate and no Loss of Attachment in Sri Lankan Laborers 14 to 46 Years of Age. J. Clin. Periodontol., Copenhagen, v.13, p.431-40, 1986.

LÖE, H.; BROWN, L.J. Early-Onset Periodontitis in the United States of America. J. Periodontol., Chicago, v.62, 608-616, 1991 .

OH, T.J.; EBER, R.; WANG, H.L. Periodontal Diseases in the Child and Adolescent. J. Clin. Periodontol., Copenhagen, v.29, p. 400-410, 2002.

PAGE, R.C. et al., Prepubertal Periodontitis. Definition of a Clinical Disease Entity. J. Periodontol., Chicago, v.54, p. $257-271,1983$.

RANNEY, R.R., Differential Diagnosis in Clinical Trials of Therapy for Periodontitis. J. Periodontol., Chicago, v.63, p. 1052-1057, 1992.

SUSIN, C; ALBANDAR, J.M. Aggressive Periodontitis in an Urban Population in Southern Brazil. J. Periodontol., Chicago, v.76, no.3, p.468-475, 2005.
TONETTI, M.S.; MOMBELLI, A. Early-Onset Periodontitis. Ann. Periodontol., Chicago, v.4, p.39-52, 1999.

VAN DER VELDEN, U. The Onset Age of Periodontal Destruction. J. Clin. Periodontol., Copenhagen, v.18, p.380-383, 1991.

WAERHAUG, J. Plaque Control in the Treatment of Juvenile Periodontitis. J. Clin. Periodontol., Copenhagen, v.4, no.1, p.29-40, 1977.

Endereço dos autores: Alex Nogueira Haas (alex_haas@uol.com.br)

Tatiana Moreno (tatimoreno_80@hotmail.com)

Cassiano Rösing (ckrosing@hotmail.com)

Rua Ramiro Barcelos, 2492 - Faculdade de Odontologia da UFRGS - Térreo Periodontia

Fone: 5191222377 - 5133165318 